\title{
An Approximation Scheme for Optimal Multicast Routing with Multiple QoS Constrains
}

\author{
Weijun Yang ${ }^{1,2}$ and Yun Zhang ${ }^{1}$ \\ ${ }^{1}$ Faculty of Automation, Guangdong University of Technology, China \\ ${ }^{2}$ Guangzhou City Polytechnic, China \\ 164331685@qq.com andywj@gcp.edu.cn
}

\begin{abstract}
This paper studies the problem of optimal multicast routing with multiple Quality-ofService (QoS) constrains (OMRMC), which is regarded as a critical component in networks. However, it is not very efficient and effective for the existing algorithms. An undirected and connected graph $G$ with $m$ edges and $n$ nodes is modeled to find a multicast routing tree from source to destination subset $D$ in this paper, and a novel approximation algorithm called APP-OMRMC is proposed for OMRMC. The theoretical validations for the proposed method are presented to show its efficiency. After that, the different networks with different types of service are evaluated. Experimental results show that the proposed method APP-OMRMC is capable of finding the optimal (or minimum-cost) multicast routing tree with the approximation factor $(1+\varepsilon)$ and time complexity of $O\left(m \tau^{K-1}\right)$.
\end{abstract}

Keywords: multicast routing, multiple QoS constrains, approximation algorithm

\section{Introduction}

Over the last decade, multicast routing (or Steiner) trees used for efficient one-to-many communications applications (e.g. web broadcasting, video teleconferencing, VOIP and HDTV) have emerged on the Internet [1-2]. Considering different quality-of-service (QoS) requirements, one of the solutions is minimum-cost multicast QoS routing. It is the problem of finding a tree in a graph from source to a subset of vertices called terminals, such that the total edge cost of which is minimal. More and more practical problems can be modeled as multicast routing tree problems nowadays, such as VLSI layout, network design, etc.

The problem of QoS multicast routing is challenging and usually known as multicast routing with multiple constrains problem (MRMC). As the best of our knowledge, finding the minimal-cost multicast routing tree with multiple QoS constrains is one of the Steiner tree problem which is NP-hard [3]. Currently, there has been much work involved in designing heuristic solutions for this problem [4-10]. Approximation algorithms for the node-weighted Steiner problem with fixed costs on each node and each edge have been presented in [11]. G. Xue et al. proposed various polynomial time algorithms [7-8] for multiple constrained optimal paths (MCOP) problem based on Yuan X's study [4]. Their algorithms resort to rounding and scaling to guarantee a solution within a factor $(1+\varepsilon)$ of the optimal solution. Other works that consider graph models with fixed edge weighs and employing the approximation algorithm for finding the optimal multicast routing tree or the optimal path can be found in [10-17].

These above mentioned researches mainly focus on solving MCOP by the efficiency approximation algorithm. Rather than previous research model, OMRMC is investigated from an approximation perspective in this paper. As far as we know, Xue's algorithm [7] is currently the fastest for path establishment in unicast routing with multiple QoS constrains. However, the solution to multicast routing problem is rarely or inefficient for the existing 
literature. In this study, a novel approximation algorithm called APP-OMRMC is proposed for OMRMC.

The rest of this paper is organized as follows. The problem to be studied is defined formally in Section 2, along with notations that will be used in later sections. In Section 3 , the approximation scheme for the optimal multicast routing with multiple QoS constrains and its theoretical analysis are presented. In Section 4, experimental results obtained from different kinds of networks are reported to verifying the algorithm. Section 5 draws the conclusion of this paper.

\section{Preliminaries}

\subsection{Problem Formulation}

A communication network with $\mathrm{K}$ QoS constraints can be represented by a connected graph $G(\mathrm{~V}, \mathrm{E}, \mathrm{W}, \mathrm{L})$, where $\mathrm{V}$ is the set of $\mathrm{n}$ vertices, $\mathrm{E}$ is the set of $\mathrm{m}$ edges, $\mathrm{W}$ is the set of weights and $\mathrm{L}$ is the set of constrains. Each edge has $K(K \geq 2)$ weights, and $W=\left\{w_{i}(\mathrm{e})\right\}, w_{i}(\mathrm{e}) \geq 0$ is the kth weight of edge $\mathrm{e}(\forall e \in E, 1 \leq i \leq K), \quad L=\left(L_{1}, L_{2}, \ldots, L_{K}\right)$ be the $\mathrm{K}$ constraints. Then, it has the following definitions.

Definition 1. MRMC (Multicast Routing with Multiple Constrains Problem). Consider an undirected graph $G(\mathrm{~V}, \mathrm{E}, \mathrm{W}, \mathrm{L})$, Let $s \in V$ be the source node and $D \subseteq V(\mathrm{~s} \notin \mathrm{D})$ be the set of terminal nodes. Denote $T_{j}(\mathrm{~s}, \mathrm{D}), j \in N$ as a multicast routing tree from source s to destination subset $\mathrm{D}$ in $G$, such that $\mathrm{T}_{j}$ is a sub-graph of $G$. Denote $w_{i}\left(\mathrm{~T}_{j}\right)$,s.t. $2 \leq \mathrm{i} \leq \mathrm{K}$ as the sum of the ith weight on edges along multicast tree $\mathrm{T}_{j}$. It is to find a multicast routing tree $\mathrm{T}_{j}$ from $\mathrm{s}$ to $\mathrm{D}$ for MRMC, such that $w_{i}\left(\mathrm{~T}_{j}\right) \leq L_{i}$.

It is said to be a feasible multicast routing tree for $\mathrm{T}_{j}$ s.t. $w_{i}\left(\mathrm{~T}_{j}\right) \leq L_{i}$. The entire feasible multicast routing tree in $G(\mathrm{~V}, \mathrm{E}, \mathrm{W}, \mathrm{L})$ are denoted to be $\left\{\mathrm{T}_{j}\right\}$.

Definition 2. OMRMC (Optimal Multicast Routing with Multiple Constrains Problem). It is to find an optimal multicast routing $T^{*}$ among feasible paths $\left\{\mathrm{T}_{j}\right\}$ in $\mathrm{G}$ for OMRMC from s to $\mathrm{D}$ and the smallest value of $\theta \in(0,1]$ such that $w_{i}\left(\mathrm{~T}^{*}\right) \leq \theta \cdot \mathrm{L}_{i}$.

Definition 3. $\alpha$-Approximation Algorithm $(\alpha \geq 1)$. An algorithm is a $\alpha$-approximation algorithm for OMRMC if the algorithm generates a multicast routing tree $T^{\text {opt }}$ from $\mathrm{s}$ to $\mathrm{D}$ such that $w_{i}\left(T^{o p t}\right) \leq \alpha \cdot \theta \cdot \mathrm{L}_{i}$, the running time of the algorithm is bounded by a polynomial in the input size of the instance as well as $1 / \alpha$.

\subsection{Deterministic Algorithm with Auxiliary Graph}

Xue et al. [7] provided an important methodology in their works, which is constructing an auxiliary graph. It transforms from an undirected graph $G$ to a directed graph $G^{K, \tau}$. Each vertex $v \in G$ is associated with $(1+\tau)^{K-1}$ vertices (since each weight has been normalized $w_{i}(\mathrm{e}) / L_{i}$, it could regard $\tau=\lceil(n-1) / \varepsilon\rceil$ ( $\varepsilon$ is the approximation factor) as the maximum integer of all constraints.) in $G^{K, \tau}$, i.e., $\mathrm{P}\left(\mathrm{u}, C_{2}, \ldots, C_{i}\right)$ and $C_{i} \in[0, \tau]$ are used for recording the first weight and the ith weight of path length from source $\mathrm{s}$ to node $\mathrm{u}$ respectively, where $2 \leq i \leq K$. To each undirected edge (u, v) in $\mathrm{G}$, it constructs the directed edge in $G^{K, \tau}$ from $\mathrm{P}\left(\mathrm{u}, C_{2}, \ldots, C_{i}\right)$ to $\mathrm{P}\left(v, D_{2}, \ldots, D_{i}\right)$ and $D_{i}=C_{i}+w_{i}(\mathrm{u}, \mathrm{v})$. Note that the infeasible paths of latter K-1 metrics have been filtered. Therefore, an optimal path in G corresponds to an optimal path from $\mathrm{P}(\mathrm{s}, 0, \ldots, 0)$ to $\mathrm{P}(\mathrm{t}, \tau, \ldots, \tau)$ in $G^{K, \tau}$.

It uses the space to trade for the time for the basic idea behind Xue's algorithm. As far as we know, for path calculation in multiple constrained QoS routing, Xue's approximation 
scheme with time complexity of $O\left(m \tau^{K-1}\right)$ is currently the fastest one. As it observed from the examples, with the Xue's deterministic algorithm with auxiliary graph, it is feasible and meaningful to seek an effective and efficient algorithm to approximate the optimal multicast routing with multiple constrains.

\section{The Proposed APP-OMRMC}

\subsection{An Approximation for Optimal Multicast Routing with Multiple Constrains}

The detailed steps of APP-OMRMC are presented as below. Algorithm APP-OMRMC

Input: $\quad G(\mathrm{~V}, \mathrm{E}, \mathrm{W}, \mathrm{L})$

Output: $T^{\text {opt }}$

source node s and destination nodes set $\mathrm{D}$, and the approximation ratio $\varepsilon$

Step 1:

For each $e \in E$ in $G(\mathrm{~V}, \mathrm{E}, \mathrm{W}, \mathrm{L})$, compute the new weights $w_{i}^{N}(\mathrm{e})=\left\lfloor\frac{w_{i}(\mathrm{e})}{L_{i}} \cdot \gamma\right\rfloor$, $i=1, \ldots, K$ and set $\tau=L_{1}^{N}=\ldots=L_{K}^{N}=\lceil\gamma\rceil, \gamma=\frac{n-1}{\varepsilon}>0$ such that $G(\mathrm{~V}, \mathrm{E}, \mathrm{W}, \mathrm{L})$ is turned to be $G^{N}\left(\mathrm{~V}, \mathrm{E}, \mathrm{W}^{N}, \tau\right)$;

\section{Step 2:}

Extend the graph $G^{N}\left(\mathrm{~V}, \mathrm{E}, \mathrm{W}^{N}, \tau\right)$ to the directed $G_{K}^{N}\left(\mathrm{~V}_{K}^{N}, \mathrm{E}_{K}^{N}, \mathrm{~W}_{K}^{N}, \tau\right)$ with vertex set $\mathrm{V}_{N}^{K}$ and edge set $E_{K}^{N}$. To a given vertex $u \in V$ is extended to a vertex group $\mathrm{P}\left(\mathrm{u}, C_{2}, \ldots, C_{i}\right)$, where $0 \leq C_{i} \leq \tau, 2 \leq i \leq K$. To a given edge (u, v) $\in \mathrm{E}, E_{K}^{N}$ contains direct edge from vertex $\mathrm{P}\left(\mathrm{u}, C_{2}, \ldots, C_{i}\right)$ to vertex $\mathrm{P}\left(v, \lambda_{2}, \ldots, \lambda_{i}\right)$, such that $\lambda_{i}=C_{i}+w_{i}^{N}(\mathrm{u}, \mathrm{v})$. All such edges have the same length $w_{1}^{N}(\mathrm{u}, \mathrm{v})$. In addition, $E_{K}^{N}$ contains zero-length edges from $\mathrm{P}\left(\mathrm{u}, C_{2}, \ldots, C_{i}\right)$ to $\mathrm{P}\left(\mathrm{u}, C_{2}+1, \ldots, C_{i}\right)$;

\section{Step 3:}

Let $\mathrm{A}\left(v, C_{2}, \ldots, C_{i}\right)$ be the prior adjacent node to $\mathrm{P}\left(v, C_{2}, \ldots, C_{i}\right)$ in multicast routing tree $T^{\text {opt }}$. Initialize the total variables as follow.

For every node $v \in V$ and $C_{i}=0$ to $\tau, 2 \leq i \leq k$ do

$$
\begin{aligned}
& \mathrm{P}\left(v, C_{2}, \ldots, C_{i}\right) \leftarrow \infty ; \mathrm{P}\left(v, C_{2}, \ldots, C_{i}\right) \leftarrow 0 ; \\
& A\left(v, C_{2}, \ldots, C_{i}\right) \leftarrow N U L L ; T^{o p t} \leftarrow\{\mathrm{s}\} ;
\end{aligned}
$$

\section{Step 4:}

for every adjacent node $v\left(v \notin T^{o p t}\right)$ of node $\mathrm{u}$, where $u \in T^{o p t}, v \notin T^{o p t}$ and $C_{i} \leq \tau$ do

if $\mathrm{P}\left(v, D_{2}, \ldots, D_{i}\right)>\mathrm{P}\left(u, C_{2}, \ldots, C_{i}\right)+\mathrm{w}_{1}(\mathrm{u}, \mathrm{v})$ where $D_{i}=C_{i}+\mathrm{w}_{i}(\mathrm{u}, \mathrm{v}), 2 \leq \mathrm{i} \leq \mathrm{K}$

then $\mathrm{P}\left(v, D_{2}, \ldots, D_{i}\right) \leftarrow \mathrm{P}\left(u, C_{2}, \ldots, C_{i}\right)+\mathrm{w}_{1}(\mathrm{u}, \mathrm{v})$

$$
\begin{aligned}
& A\left(v, D_{2}, \ldots, D_{i}\right) \leftarrow u \\
& T^{o p t} \leftarrow \text { node } \mathrm{v} \text { and edge }(\mathrm{u}, \mathrm{v}) ;
\end{aligned}
$$

\section{end if}

\section{Step 5:}

if $w_{1}^{N}\left(\mathrm{~T}^{o p t}\right)>\tau$

\section{Step 6:}

then return No feasible multicast routing tree, Exit;

else return $T^{\text {opt }}$ by omitting the later K-1 components of each extended node on the finding multicast routing tree;

end if 


\subsection{The Analysis of APP-OMRMC}

Theorem 1. APP-OMRMC obtains a feasible multicast routing $T^{\text {opt }}$ from source $\mathrm{s}$ to destination set $\mathrm{D}$ which minimizes $\max _{1 \leq i \leq K} w_{i}^{N}\left(T_{j}\right)$ among all multicast routing trees in $G_{K}^{N}\left(\mathrm{~V}_{K}^{N}, \mathrm{E}_{K}^{N}, \mathrm{~W}_{K}^{N}, \tau\right)$. The worst case time complexity of the algorithm is $O\left(m \tau^{K-1}\right)$.

Proof. Since the Step 4 searches $\mathrm{P}\left(v, D_{2}, \ldots, D_{i}\right)$ in lexicographic order for every adjacent node $v\left(v \notin T^{o p t}\right)$ of node $\mathrm{u}\left(u \in T^{o p t}\right)$ in algorithm APP-OMRMC, and guarantees $D_{i}$ is not more than the largest integer $\tau$, which means that APP-OMRMC searches a shortest path in the space of $\max _{1 \leq i \leq K} w_{i}^{N}\left(T_{j}\right)$, therefore APP-OMRMC obtains a multicast routing tree $T^{\text {opt }}$ from source to destination set $D$ that minimizes $\max _{1 \leq i \leq K} w_{i}^{N}\left(T_{j}\right)$ among all multicast routing trees in $G_{K}^{N}$.

During the running of APP-OMRMC, $G_{K}^{N}$ have $O\left(\mathrm{n} \cdot Q^{K-1}\right)$ vertices and $O\left(2 m \cdot Q^{K-1}+n \cdot Q^{K-1}\right)$ edges, which confirm our observation that the space complexity is fairly large. Moreover, $G_{K}^{N}$ contains a directed edge from $\mathrm{P}\left(u, C_{2}, \ldots, C_{i}\right)$ to $\mathrm{P}\left(v, D_{2}, \ldots, D_{i}\right)$ with length $w_{i}(\mathrm{u}, \mathrm{v})$. Therefore, the optimal multicast routing tree $T^{\text {opt }}$ found in APP-OMRMC minimizes $\max _{1 \leq i \leq K} w_{i}^{N}\left(T_{j}\right)$ among multicast routing trees in $G_{K}^{N}$.

Since each $w_{i}(e)$ is a positive real-value, for $2 \leq i \leq \mathrm{K}, e \in E$, the existence of directed edge from $\mathrm{P}\left(u, C_{2}, \ldots, C_{i}\right)$ to $\mathrm{P}\left(v, D_{2}, \ldots, D_{i}\right)$ in $G_{K}^{N}$ implies that $C_{i}<D_{i}$ for $2 \leq i \leq \mathrm{K}, e \in E$. Therefore, the graph $G_{K}^{N}$ is acyclic. For an acyclic graph, the worst case time complexity of each running process is $O\left(2 m \cdot \tau^{K-1}+n \cdot \tau^{K-1}\right)=O\left(m \tau^{K-1}\right)$. So the worst case time complexity of APPOMRMC is $O\left(\mathrm{~m} \tau^{K-1}\right)$.

According to Theorem 1, it concludes that the time complexity of APP-OMRMC is nearly the same as that of Xue-based scheme in finding the MCOP [7], but it is easier to implement according to the procedure of algorithm APP-OMRMC.

Theorem 2. To any feasible multicast routing tree $\mathrm{T}_{j}$ in $G$, it is a feasible multicast routing tree in $G_{K}^{N}$.

Proof. To a feasible multicast routing tree $\mathrm{T}_{j}$ in $G$, it has

$$
w_{i}\left(\mathrm{~T}_{j}\right)=\sum_{e \in \mathrm{T}_{j}} w_{i}(\mathrm{e}) \leq L_{i}
$$

It implies that

Since

$$
\frac{\sum_{e \in \mathrm{T}_{j}} w_{i}(\mathrm{e})}{L_{i}} \cdot \gamma=\sum_{e \in \mathrm{T}_{j}} \frac{w_{i}(\mathrm{e})}{L_{i}} \cdot \gamma \leq \gamma
$$

And

$$
w_{i}^{N}(\mathrm{e})=\left\lfloor\frac{w_{i}(\mathrm{e})}{L_{i}} \cdot \gamma\right\rfloor \leq \frac{w_{i}(\mathrm{e})}{L_{i}} \cdot \gamma
$$

$$
\gamma \leq\lceil\gamma\rceil=\tau
$$

Then it has

$$
\begin{aligned}
w_{i}^{N}\left(\mathrm{~T}_{j}\right)= & \sum_{e \in \mathrm{T}_{j}} w_{i}^{N}(\mathrm{e}) \\
& =\sum_{e \in \mathrm{T}_{j}}\left\lfloor\frac{w_{i}(\mathrm{e})}{L_{i}} \cdot \gamma\right\rfloor \\
& \leq \sum_{e \in \mathrm{T}_{j}} \frac{w_{i}(\mathrm{e})}{L_{i}} \cdot \gamma
\end{aligned}
$$

According to Equations (2), (4) and (5), it has

$$
w_{i}^{N}\left(\mathrm{~T}_{j}\right) \leq \tau
$$


Hence, any feasible multicast routing tree $\mathrm{T}_{j}$ in $G$ is a feasible multicast routing tree in $G_{K}^{N}$.

Theorem 3. Algorithm APP-OMRMC finds $\mathrm{a}(1+\varepsilon)$ approximation multicast routing tree to OMRMC.

Proof. To the optimal multicast routing tree $T^{*}$ in $G$, it has

$$
\begin{gathered}
w_{i}\left(T^{*}\right) \leq \theta \cdot L_{i} \\
w_{i}^{N}\left(T^{*}\right)=\sum_{e \in T^{*}} w_{i}^{N}(\mathrm{e})=\sum_{e \in T^{*}}\left\lfloor\frac{w_{i}(\mathrm{e})}{L_{i}} \cdot \gamma\right\rfloor
\end{gathered}
$$

This implies that

$$
\begin{aligned}
w_{i}^{N}\left(T^{*}\right) \leq & \sum_{e \in T^{*}}\left(\frac{w_{i}(\mathrm{e})}{L_{i}} \cdot \gamma\right) \\
& =\frac{w_{i}\left(T^{*}\right)}{L_{i}} \cdot \gamma \\
& \leq \theta \cdot \gamma
\end{aligned}
$$

On the other hand, $T^{o p t}$ minimizes $\max _{1 \leq i \leq K} w_{i}^{N}\left(T_{j}\right)$ among all multicast routing trees in $G_{K}^{N}$ and $T^{*}$ is a feasible multicast routing tree as mentioned in Theorem 1 and in Theorem 2 respectively, then it has

$$
\max _{1 \leq i \leq K} w_{i}^{N}\left(T^{o p t}\right) \leq \max _{1 \leq i \leq K} w_{i}^{N}\left(T^{*}\right)
$$

Since $T^{O P T}$ have $|V|-1$ hops at most, and there are $\mathrm{n}$ nodes in $\mathrm{G}$, it implies that

$$
\begin{aligned}
& w_{i}^{N}\left(T^{o p t}\right)=\sum_{e \in T^{o p t}} w_{i}^{N}(\mathrm{e})=\sum_{e \in T^{\text {opt }}}\left\lfloor\frac{w_{i}(\mathrm{e})}{L_{i}} \cdot \gamma\right\rfloor \\
& \geq \sum_{e \in T^{o p t}}\left(\frac{w_{i}(\mathrm{e})}{L_{i}} \cdot \gamma-1\right) \\
&=\sum_{e \in T^{o p t}}\left(\frac{w_{i}(\mathrm{e})}{L_{i}} \cdot \gamma\right)-\sum_{e \in T^{o p t}} 1 \\
& \geq \frac{w_{i}\left(T^{o p t}\right)}{L_{i}} \cdot \gamma-(\mathrm{n}-1)
\end{aligned}
$$

According to Equations (9), (10) and (11), it has

$$
\frac{w_{i}\left(T^{o p t}\right)}{L_{i}} \cdot \gamma-(\mathrm{n}-1) \leq \theta \cdot \gamma
$$

It implies that

$$
\frac{w_{i}\left(T^{o p t}\right)}{L_{i}} \leq \theta+\frac{\mathrm{n}-1}{\gamma}
$$

Let $\frac{\mathrm{n}-1}{\gamma}=\varepsilon \cdot \theta, \varepsilon>0$, then

$$
w_{i}\left(T^{o p t}\right) \leq(1+\varepsilon) \cdot \theta \cdot \mathrm{L}_{i}, 1 \leq i \leq K
$$

Hence, APP-OMRMC can find a $(1+\varepsilon)$ approximation multicast routing tree to OMRMC, and the time complexity of APP-OMRMC is $O\left(\mathrm{~m} \tau^{K-1}\right)$. 


\section{Simulation and Performance Evaluation}

It shall evaluate both the performance of APP-OMRMC and the performance of obtained multicast routing tree experimentally in this section. To evaluate its efficiency, APPOMRMC was implemented and conducted a performance study. Special network namely CERNet [2] is used in these experiments which are run on an Intel Core Duo CPU 1.66 GHz PC with 2 GB memory. There are 25 nodes and 30 edges in CERNet as showed in Figure 1, and other parameters for the network can be found in the network link address: http://code.google.com/p/efptas/downloads/list. Each link of CERNet has three weights, which corresponds to Cost, Delay and Jitter.

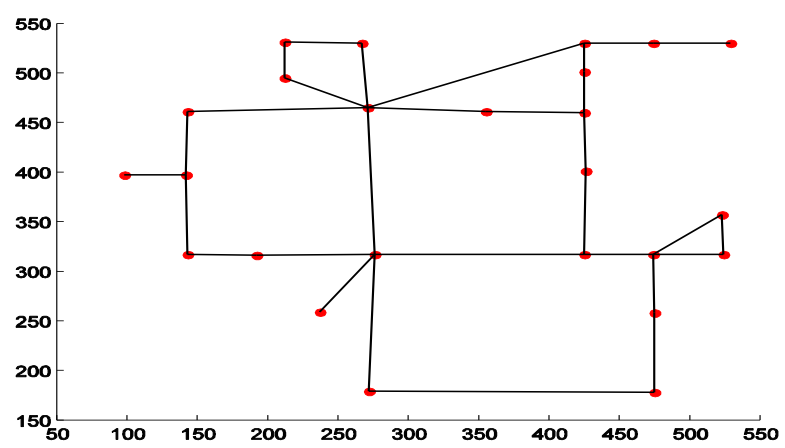

Figure 1. The Network Topology of CERNet

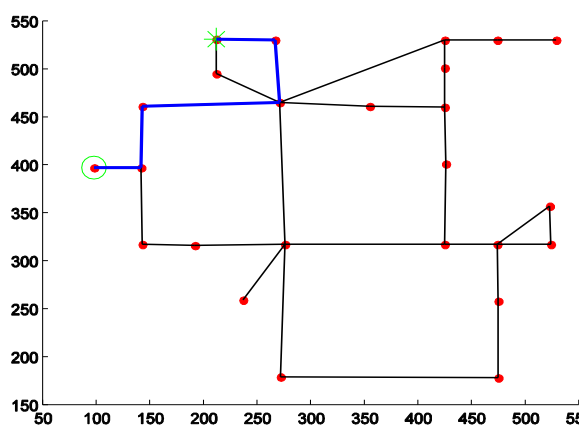

(a) The destination node No.6

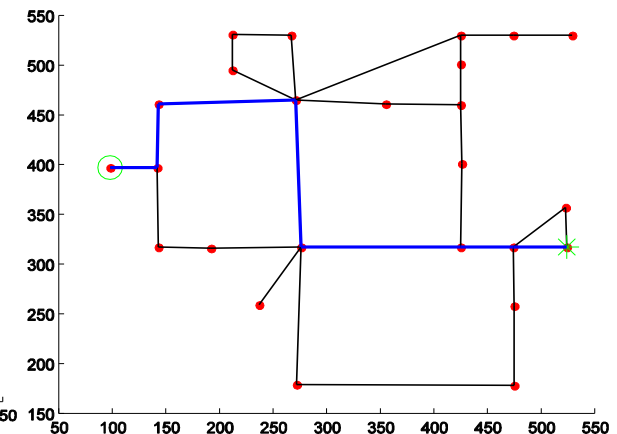

(b) The destination node No.25

Figure 2. The Minimal Cost Path

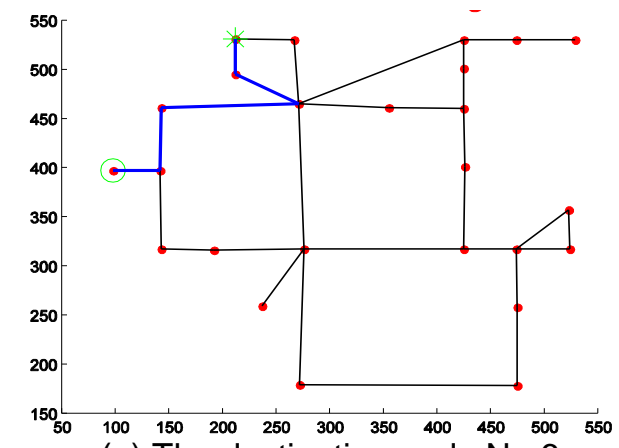

(a) The destination node No.6

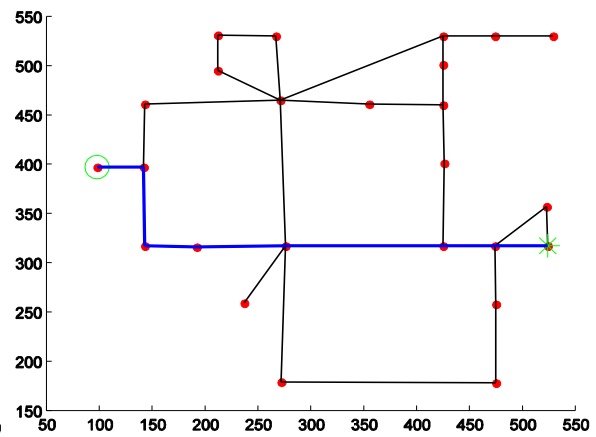

(b) The destination node No.25

Figure 3. The Minimal Delay Path 


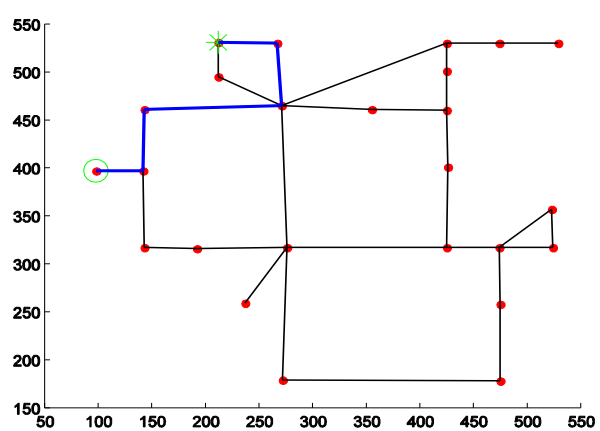

(a) The destination node No.6

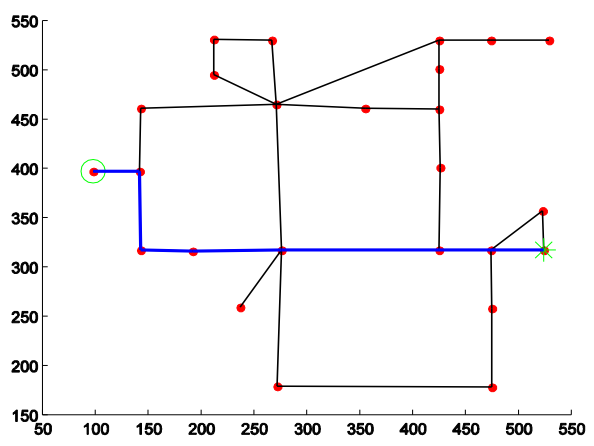

(b) The destination node No.25

Figure 4. The Minimal Jitter Path

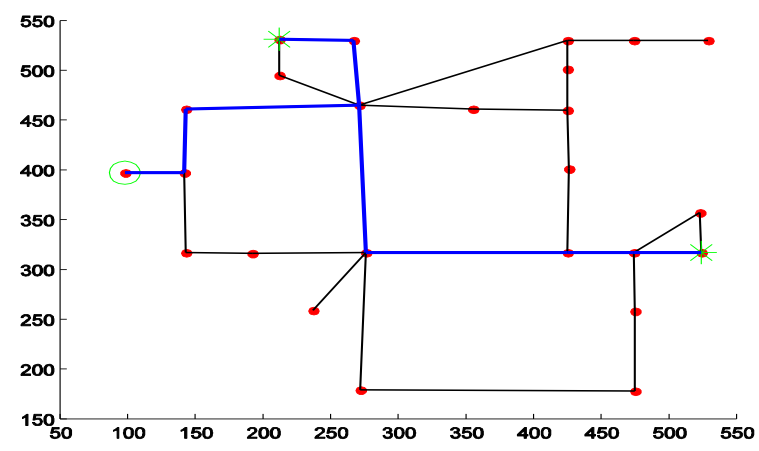

Figure 5. The Optimal Multicast Routing Tree with Three Constrains

All the red points are denoted as nodes of the network, the black wires as the links, the green circle as the source node s (No.16) and the green star as the destination node set D (No.6 and No.25) in the network CERNet respectively for Figure 2- Figure 5. The blue paths in these figures indicate the minimal or the optimal paths from source to destination. Figure 2(a) and Figure 2(b) show the minimal cost paths from source node (No.16) to destination nodes (No.16 and No.25) respectively in the network, each link of which is with the weight cost. As the same as Figure 2(a) and Figure 2(b), the minimal delay path is show in Figure 3 (a) and Figure 3(b), each link of which is with the weight delay. Figure 4 illustrates the minimal delay jitter path, and the link is with the weight delay jitter. As expected, the optimal multicast routing tree with three constrains by the algorithm APP-OMRMC is able to be found, and the optimal multicast routing tree described in Figure 5, for which the source node (No.16) and the destination node set D (No.6 and No.25), shows the corresponding results.

\section{Conclusion}

It discusses the problem of optimal multicast routing with multiple in this paper. An approximation scheme for the OMRMC (APP-OMRMC) is proposed, which is based on the technique of auxiliary graph construction, scaling and rounding. The algorithm can achieve the low complexity and obtain the approximation optimal multicast routing tree, respectively. According to the experiment on the random network, it shows that the proposed method APPOMRMC is capable of finding the optimal (or minimum-cost) multicast routing tree with the approximation factor $(1+\varepsilon)$. 


\section{Acknowledgements}

This work described in this paper is supported by Specialized Research Fund for the Doctoral Program of Higher Education (No. 20124420130001), and the Science and Technology Program of Guangzhou, China (No. 201510010192).

\section{References}

[1] Z. Zhu, S. Li and X. Chen, "Design QoS-Aware Muli-Path Provisioning Strategies for Efficient CLOUDAssisted SVC Video Streaming to Heterogeneous Clients", IEEE Transactions on Multimedia, vol. 15, no. 4, (2013), pp. 758-768.

[2] J. Huang, X. Huang and Y. Ma, "Routing with multiple quality of services constraints: An approximation perspective", Journal of Network and Computer Applications, vol. 35, no. 2, (2012), pp. 465-475.

[3] Y. Xiao, K. Thulasiraman, X. Fang, D. Yang and G. Xue, "Computing a Most Probable Delay Constrained Path: NP-Hardness and Approximation Schemes”, IEEE Transactions on Computers, vol. 61, no. 5, (2012), pp. 738-744.

[4] X. Yuan, "Heuristic algorithms for multiconstrained quality-of-service routing", IEEE/ACM Transactions on Networking, vol. 10, no. 2, (2002), pp. 244-256.

[5] S. Chen, M. Song and S. Sahni, "Two techniques for fast computation of constrained shortest paths, IEEE/ACM Transactions on Networking", vol. 16, no. 1, (2008), pp. 105-115.

[6] J. Huang and Y. Liu, "MOEAQ: A QoS-aware multicast routing algorithm for MANET", Expert Systems with Applications, vol. 37, no. 2, (2010), pp. 1391-1399.

[7] G. Xue, A. Sen, W. Zhang, J. Tang and K. Thulasiraman, "Finding a path subject to many additive QoS constraints", IEEE/ACM Transactions on Networking, vol. 15, no. 1, (2007), pp. 201-211.

[8] G. Xue, W. Zhang, J. Tang and K. Thulasiraman, "Polynomial time approximation algorithms for multiconstrained QoS routing", IEEE/ACM Transactions on Networking, vol. 16, no. 3, (2008), pp. 656-669.

[9] B. M. Waxman, "Routing of multipoint connections, IEEE Journal on Selected Areas in Communications, vol. 6, no. 9, (1988), pp. 1617-1622

[10] G. Feng and T. Korkmaz, "A Fast Hybrid \&-Approximation Algorithm for Computing Constrained Shortest Paths", IEEE Communications Letters, vol. 17, no. 7, (2013), pp. 1471-1474.

[11] H. C. Lin and H. M. Yang, "An Approximation Algorithm for Constructing Degree-Dependent NodeWeighted Multicast Trees", IEEE Transactions on Parallel and Distributed Systems, vol. 25, no. 8, (2014), pp. 1976-1985.

[12] G. Pei, S. Parthasarathy, A. Srinivasan and A. K. S. Vullikanti, "Approximation Algorithms for Throughput Maximization in Wireless Networks with Delay Constraints", IEEE/ACM Transactions on Networking, vol. 21, no. 6, (2013), pp. 1988-2000.

[13] T. Lu and J. Zhu, "Genetic Algorithm for Energy-Efficient QoS Multicast Routing”, IEEE Communications Letters, vol. 17, no. 1, (2013), pp. 31-34.

[14] Y. S. Su, S. L. Su and J. S. Li, "Joint Topology-Transparent Scheduling and QoS Routing in Ad Hoc Networks", IEEE Transactions on Vehicular Technology, vol. 63, no. 1, (2014), pp. 372-389.

[15] T. H, Szymanski, "Max-Flow Min-Cost Routing in a Future-Internet with Improved QoS Guarantees", IEEE Transactions on Communications, vol. 61, no. 4, (2013), pp. 1485-1497.

[16] X. Fang, D. Yang and G. Xue, "MAP: Multiconstrained Anypath Routing in Wireless Mesh Networks", IEEE Transactions on Mobile Computing, vol. 12, no. 10, (2012), pp. 1893-1906.

[17] D. Huijun, Q. Hua and Z. Jihong, "QoS routing algorithm with multi-dimensions for overlay networks", China Communications, vol. 10, no. 10, (2013), pp. 167-176.

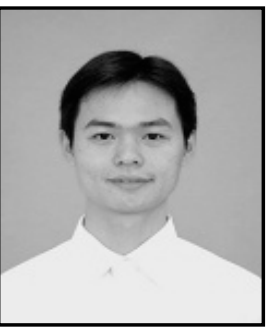

\section{Authors}

Weijun Yang, he received the B.S. and M.S. degrees in information engineering from Guangdong University of Technology, Guangzhou, China, in 2005 and 2008, respectively, where he is currently working toward the Ph.D. degree in control theory and control engineering. His research interests include optimal control, network systems and signal processing. 


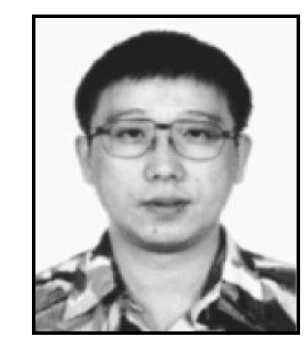

Yun Zhang, he received the B.S. and M.S. degrees in automatic engineering from Hunan University, Changsha, China, in 1982 and 1986, respectively, and the Ph.D. degree in automatic engineering from the South China University of Science and Technology, Guangzhou, China, in 1998. He is currently a Professor with the Department of Automation, Guangdong University of Technology, Guangzhou, China. His research interests include intelligent control systems, network systems, and signal processing. 
International Journal of Future Generation Communication and Networking Vol. 9, No.9, (2016) 